\title{
Single-step method for fiber-optic probe-based full-range spectral domain optical coherence tomography
}

\author{
Eun Jung Min, ${ }^{1}$ Jun Geun Shin, ${ }^{1}$ Jae Hwi Lee, ${ }^{1}$ Yoshiaki Yasuno, ${ }^{2}$ \\ and Byeong $\mathrm{Ha} \mathrm{Lee}{ }^{1, *}$ \\ ${ }^{1}$ School of Information and Communications, Gwangju Institute of Science and Technology, \\ 261 Cheomdan-gwagiro, Buk-gu, Gwangju 500-712, South Korea \\ ${ }^{2}$ Institute of Applied Physics, University of Tsukuba, Tennôdai 1-1-1, Tsukuba, Ibaraki 305-8573, Japan \\ ${ }^{*}$ Corresponding author: leebh@ gist.ac.kr
}

Received 29 January 2013; revised 12 April 2013; accepted 21 June 2013;

posted 21 June 2013 (Doc. ID 184270); published 15 July 2013

\begin{abstract}
We propose a single-step method appropriated for a fiber-optic probe-based full-range spectral domain optical coherence tomography (OCT). The fiber-optic probe was scanned over a sample with a magnetically driven actuator. In the reference arm, a phase shift of $\pi / 2$ was applied during two neighbor axial scanning, from which the complex spectral interferogram was directly reconstructed. Since the complexconjugate-free OCT image is obtained by doing just one Fourier transform on the complex interferogram, obtaining the full-range image is simple in algorithm and effective in computation time. Some full-range images of biological samples created with the proposed method are presented and the processing time is analyzed. (C) 2013 Optical Society of America

OCIS codes: (110.4500) Optical coherence tomography; (110.2650) Fringe analysis; (120.5060)

Phase modulation; (100.0100) Image processing.

http://dx.doi.org/10.1364/AO.52.005143
\end{abstract}

\section{Introduction}

Optical coherence tomography (OCT) is a powerful noninvasive imaging modality that provides highresolution tomographic images. As its original form, time-domain OCT is time consuming and mechanically unstable because it requires a moving part for axial scans (A-scan) [1]. The second-generation, spectral domain OCT (SD OCT) [2-4] operates at a higher speed and shows improvement in sensitivity. However, it leads to the inevitable problem of overlapping real and complex-conjugate images. The fast Fourier transform (FFT) of an interferogram is symmetric with respect to the interferometer's zerophase delay, which reduces the depth range by half.

$1559-128 \mathrm{X} / 13 / 215143-09 \$ 15.00 / 0$

(C) 2013 Optical Society of America
Therefore, in order to remove the complex-conjugate artifact, various full-range methods have been proposed. They can be categorized into the multi- and single-frame methods. As the multi-frame method, the phase shifting [ $\underline{5}-10]$ and the simultaneous multiple detection method $[11,12]$ were researched. For the single-frame method, the BM scan [13] (B-scan: lateral scan, M-scan: reference mirror scan), the B-scan-based Hilbert transform reconstruction [14], and the phase shift between two A-lines [15] methods have been proposed.

The phase-shifting method is the most common way for retrieving a complex image from a few frames of interferograms having different phases at the reference beam. However, it requires several interferograms at the same location to build a single complex A-line, which is time consuming; the two-step phase-shifting method has been adopted particularly 
because it demands just two interferograms $[6,7,9,10]$. By utilizing multiple spectrometers [11] or a $3 \times 3$ coupler [12] for parallel detections, two interferograms of a phase difference could be simultaneously captured. Even though this technique has the advantage of phase stability against the sample motion and external environmental perturbations, the system either expands or requires additional devices. Additionally, it has unwanted power loss.

Recently, the single-frame full-range method has been widely researched, in which the complex image is obtained while modulating the phase of the reference beam during the lateral sample scanning. In this case, the complex-conjugate image can be eliminated without giving up the measurement time. Among the single-frame full-range methods, the Yasuno's BM scan [13] and Wang's Hilbert transform [14] methods in particular have been employed by many groups [16-20]. In practical realization, the phase-shifting method using an off-axis galvano scanner, without any phase shifting in the reference arm, was developed [16-18]. However, the BM scan and the Hilbert transform method require as much as triple FFT processes. In order to reduce the process time, the coupled equation based on the phase shift between neighboring A-scans has been proposed [15]. Although it conducts only a single FFT, it requires an additional calculation after the FFT.

OCT systems based on fiber-optic endoscopic and handheld probes [19-21] have been developed for preclinical and clinical researches. They have the advantages of compactness, flexibility, alignment free, high-speed scanning, and particularly good compatibility with other fiber-based systems. Similar to the conventional bulk-optic-based system, the probebased SD OCT system also presents the complexconjugate artifact problem. Interestingly, studies of a probe-based full-range system $[19,20]$ remain rare, despite its potential for high-contrast internal organ imaging. However, some full-range methods applied for the bulk-optic-based system are not suitable for the fiber-optic probe-based system. For example, receiving several interferograms at a same position, of different phases, is not simple with a fiber-optic scanner. By taking these limits into consideration, the single-frame full-range method would be more suitable for a fiber-optic probe-based OCT system since it can have the phase shift and the sample scanning simultaneously.

In this paper, we adopt and modify the two-step phase-shifting method for a full-range fiber-optic probe-based system by considering factors, such as a time- and space-efficient image processing algorithm. In the original two-step phase-shifting process, two successive A-line measurements are made at a same location but with a phase shift of $\pi / 2$, to extract the complex spectral interferogram for a full-range image. In our modified method, the complex spectral interferogram is constructed with two neighboring A-lines and the full-range image is obtained with a single FFT process, the so-called single-step method in this paper. The main content is composed of largely two parts. First, the singlestep method is applied to a bulk-optic-based system and for confirming the concept of the proposed method. Second, the method is applied to the system equipped with the fiber-optic probe fabricated for this experiment. The complex-conjugate-free, fullrange SD OCT images obtained with both bulkand fiber-optic probe-based systems are presented. Finally, the computation times of the standard (real FFT in wavenumber domain), the BM scan, and the single-step method are compared and analyzed.

\section{Single-Step Method Confirmed with Bulk-Optic-Based System}

\section{A. Schematic and Method Introduction}

A bulk-optic-based SD OCT system was constructed with the scheme of Fig. 1 to confirm the concept of the single-step method. For the SD OCT system, a broadband light source having a center wavelength of $840 \mathrm{~nm}$ and a spectral bandwidth of $62 \mathrm{~nm}$ (Superlum SLD-35-HP) was used. The light was split into reference and sample beams using a 50/50 optical fiber coupler. The sample beam was reflected or scattered from the sample under investigation, and the reference beam was reflected from a reference mirror. In the sample arm, an off-axis galvano scanner was utilized for both the phase shift and the sample scanning. The beams from the sample and the reference arms combine through the coupler and interfere with each other. Finally, the combined beam was delivered to a spectrometer and captured by a line-scan CCD camera (Atmel Aviiva SM2 CL 2014).

The spectral interferogram at the line-scan CCD is mathematically given as

$$
\begin{aligned}
I(\omega, x)= & I_{R}(\omega)+I_{s}(\omega, x) \\
& +2\left[I_{R}(\omega) I_{s}(\omega, x)\right]^{1 / 2} \cos \left(\Delta \varphi_{s}(\omega, x)+\varphi(x)\right),
\end{aligned}
$$

where $\omega$ and $x$ are the optical angular frequency and the lateral position, respectively. $I_{R}(\omega)$ and $I_{s}(\omega, x)$ are the light intensities of the reference and the

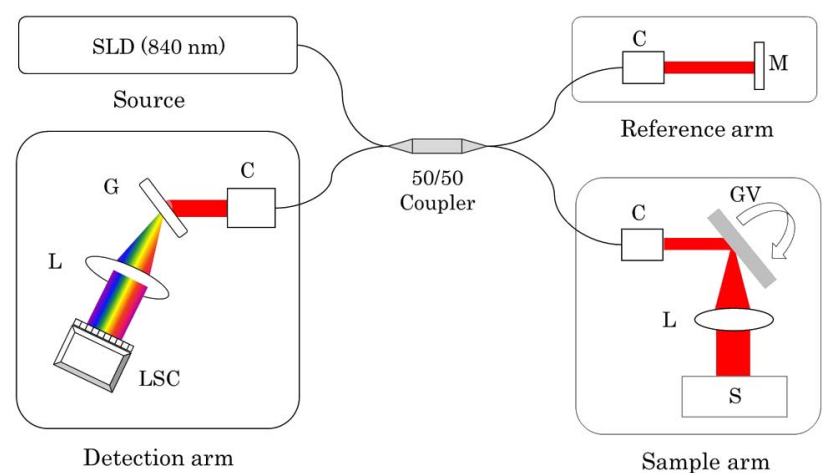

Fig. 1. Schematic of a full-range SD OCT system. SLD, superluminescent diode; $\mathrm{C}$, collimator lens; $\mathrm{M}$, mirror; L, lens; LSC, line scan CCD camera; G, grating; GV, galvano scanner; and S, sample. 
sample beams, $\Delta \varphi_{s}(\omega, x)$ is the lateral positiondependent phase delay of the sample beam relative to the reference beam, and $\varphi(x)$ is the lateral position-dependent phase shift that occurs during sample scanning. Generally, in order to get a complex interferogram, $\varphi(x)$ is adjusted with a proper phase shift. The experimental axial and lateral resolutions were both $10 \mu \mathrm{m}$. The lateral resolution was measured by plotting the intensity profile of the beam reflected from a sharp edge-cleaved silicon wafer; $20-80 \%$ width of the measured intensity was regarded as the spot size of the beam [22]. Seven A-line scans were made within the lateral spot size of the sample beam (oversampling ratio $=7$ ). Thus, we could assume that only the externally applied phase shift was effective when we consider the phase difference between two neighboring A-lines. Or the phase shift due to the sample surface variation could be ignored in a similar fashion when calculating the Doppler shift in Doppler OCT [23-25].

Figure 2 illustrates the schematic of the off-axis galvano scanner that gives the phase shift to the sample beam while sample scanning, which is necessary to construct a complex spectral interferogram. Labels GV, PA, a, and $\theta$ represent the galvano scanner, pivot axis, galvano mirror offset, and maximum scan angle, respectively. $n, I_{n}$, and $I_{n+1}$ are the consecutive number of A-lines in the lateral direction, and spectral interferograms taken at $n$th and

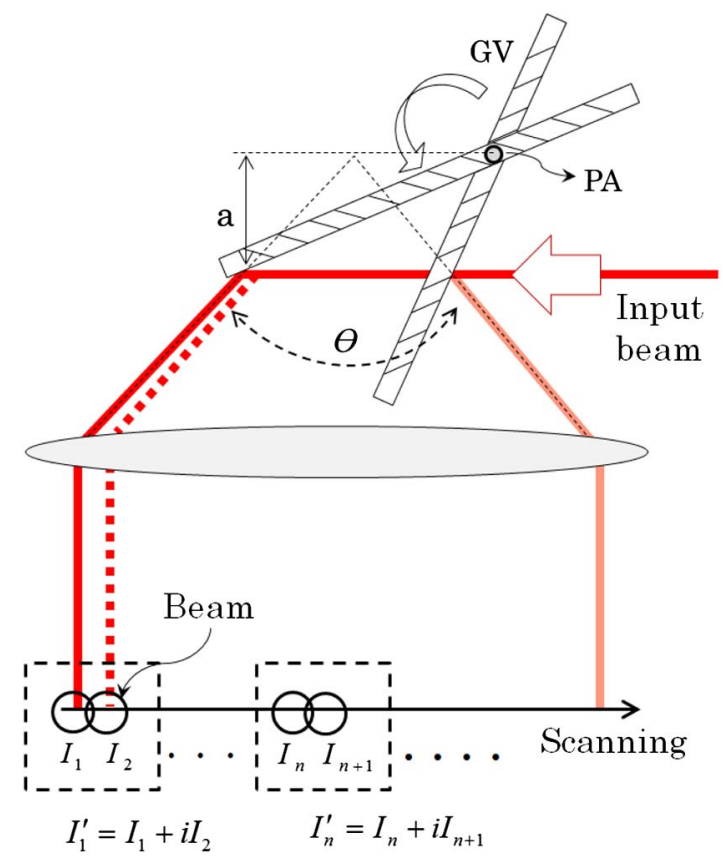

Fig. 2. Illustration showing the concept of the suggested singlestep method in a bulk-optic-based system. An off-axis galvano scanner in the sample arm was used for phase shift. $n$, number of A-lines; $I_{n}$ and $I_{n+1}$, two neighboring A-lines at $n$th and $n+1$ th in lateral direction; $\mathrm{GV}$, galvano scanner; PA, pivot axis; a, galvano mirror offset; $\theta$, maximum scan angle. A pair of neighboring A-lines is used to construct complex spectral interferogram, which is required for eliminating complex-conjugate term after performing FFT in the wavenumber domain. $(n+1)$ th lateral positions. When the phase shift of $\Delta \varphi=\pi / 2$ is applied between two neighboring A-lines, the neighboring spectral interferograms are expressed as

$$
I_{n}=I(\omega), \quad I_{n+1}=I(\omega, \Delta \varphi=\pi / 2) .
$$

Here, the two signals $I_{n}$ and $I_{n+1}$ have the same amplitudes but the phases are different by $\pi / 2$. Thus, the complex spectral interferogram $\tilde{I}_{n}$ for a full-range image can be mathematically constructed as a simple combination of two signals:

$$
\tilde{I}_{n}=I_{n}+i I_{n+1}=I(\omega)+i I(\omega, \Delta \varphi=\pi / 2) .
$$

With this complex interferogram, the complexconjugate-free time-domain signal is obtained by performing a single inverse FFT:

$$
\tilde{f}(\tau)=\mathrm{FFT}^{-1}\left\{\tilde{I}_{n}(\omega)\right\}=\sum_{m} \Gamma\left[\tau+\left(\tau_{r}-\tau_{m}\right)\right] .
$$

The figures $\tau_{r}$ and $\tau_{m}$ represent the traveling times from the reference mirror and from the position of a layer $m$ in the sample, respectively. Their difference, $\tau_{r}-\tau_{m}$, is proportional to the optical path length difference between the reference and the sample arms.

\section{B. Performance}

In an off-axis galvano scanner, when the maximum scanning angle $\theta$ is assumed small enough, the phase shift at the $n$th position in the lateral direction is calculated as [17]

$$
\varphi_{n}=\left(\frac{4 \pi a \theta}{N \lambda}\right) n
$$

where $N$ is the total number of A-lines and $\lambda$ is the operating wavelength. The equation says that the phase increases proportionally to the number of Alines $n$, thus to the position in the lateral direction.

By adjusting the parameters of Eq. (5) in experiments, the phase interval of $\pi / 2$ could $\bar{b}$ e obtained. At first, with an infrared alignment card (Thorlabs VRC2D05) as a sample, 2000 A-line spectral interferograms had been captured during a single sample scan. With each interferogram, FFT was performed and its phase was extracted. And then phase shift occurs along a lateral line was plotted in Fig. 3 and fitted with a linear curve. The slope of the fitted curve means the phase difference between two neighboring A-lines. To have the phase interval of $\pi / 2$, fine adjustment was made mainly with the galvano mirror offset $a$. The curve fitting in Fig. 3 says that the phase interval is about $1.57 \mathrm{rad}$, which is very close to the necessary one of $\pi / 2$. In the experiment, the parameters a, $\theta, N$, and $\lambda$ were adjusted to be $3.0 \mathrm{~mm}, 4^{\circ}, 2000$, and $840 \mathrm{~nm}$, respectively.

With a flat mirror as a sample, the interferograms of one B-scan were captured and the proposed 


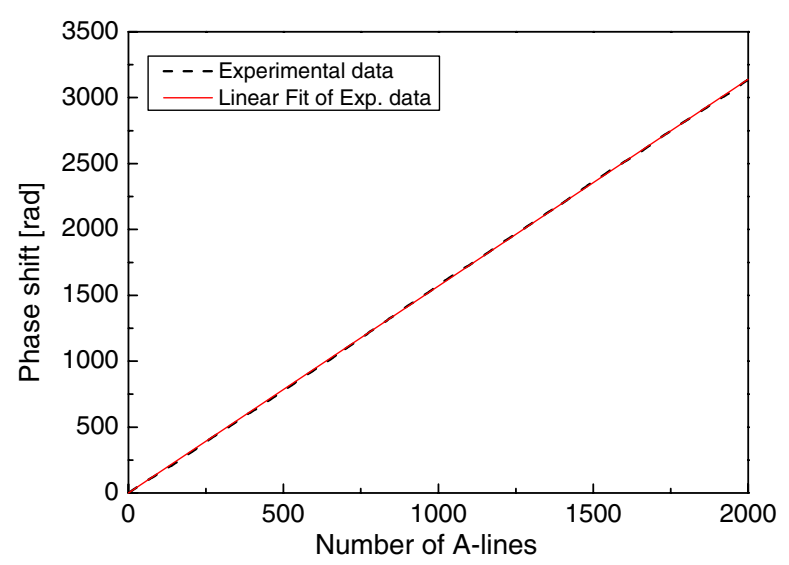

Fig. 3. Graph of phase shift with respect to the number of A-lines (lateral position) in radian units. The phase increases linearly with respect to the number of A-lines, and the slope of fitting function is about $1.57 \mathrm{rad}$, which corresponds to phase shift between two neighboring A-lines.

single-step method was applied to a pair of neighboring A-line interferogram signals. Figure 4 shows the FFT spectrum of the complex interferogram constructed by using Eq. (3). We can see that the complex-conjugate artifact signal (small peak on the left) was highly suppressed; otherwise it had the same intensity as the wanted signal (big peak on the right). The suppression ratio defined as the ratio of the two Fourier peaks [16] was measured as high as $-44 \mathrm{~dB}$.

\section{Image Reconstruction Processing}

With a gemstone pearl [26], the SD OCT images have been reconstructed by using three methods and comparing their complexities and processing times; the standard method having no full-range capability, the conventional BM scan method, and the proposed single-step method. First of all, a two-dimensional (2D) spectral interferogram of Fig. 5(a) was formed with the interferograms of a single $\overline{\mathrm{B}-\mathrm{scan}}$, obtained while modulating the phase with the slop of Fig. 3 . The vertical line at a position $x$ is the A-line interferogram, taken at that particular lateral position and transformed into the optical wavenumber $k$ domain.

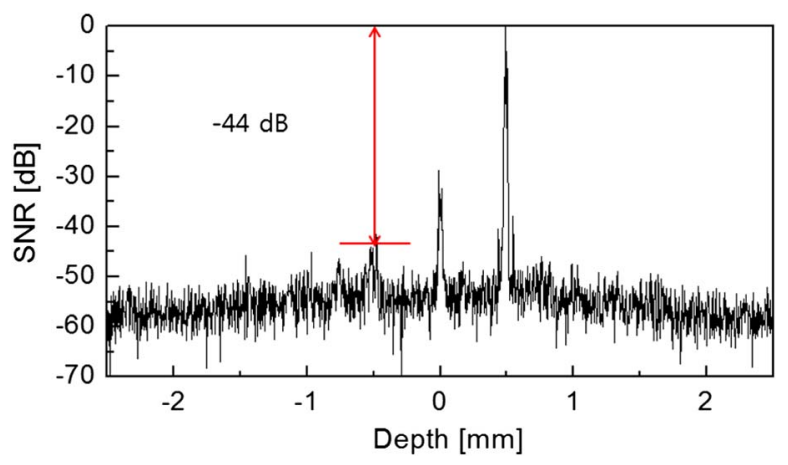

Fig. 4. Graph representing the complex-conjugate artifact suppression ratio in a single A-line profile. The suppression ratio is $-44 \mathrm{~dB}$.
At first, the standard SD OCT image of Fig. 5(e) was obtained by performing FFT on each $k$ interferogram. As expected, it shows the symmetrically overlapped image, folded with respect to the interferometer's zero-delay line, thereby making the image difficult to distinguish. Second, the BM scan method was performed, in which the interferogram was processed by the FFT in the lateral direction first. Figure 5(b) is the FFT spectrum for a single lateral line, represented in Fig. 5 (a) as the horizontal dotted line. It has two Fourier peaks, located symmetrically with respect to the zero spatial frequency, which means the interferogram [the one taken along the horizontal line in Fig. 5(a)] has a single spatial frequency. One of the Fourier peaks was selected by using a spatial frequency filter [the trapezoidshape solid line in the left of Fig. 5(b)] and then inverse Fourier transformed, which becomes the complex interferogram. Following on from applying the process for all wavenumbers, the $2 \mathrm{D}$ complex interferogram of Fig. 5(c) was obtained. The full-range image of Fig. 5(d) was subsequently achieved by performing FFT in the vertical direction again or in the $k$ domain. Finally, the single-step method was performed, which gave the complex-conjugate-free full-range SD OCT image of Fig. 5(f); it required only a single FFT process in the $k$ domain.

From the feature point of view, Fig. 5(f) is well matched with Fig. 5(d) obtained with the BM scan method. However, we can see that DC and autocorrelation terms, not related with the phase shift between A-lines in general, were not eliminated in Fig. 5(f). Although presenting limitation, the single-step method is still attractive since by adding a simple image processing, we can remove the nonphase shift-related signals in some cases [10]. It is noteworthy that in Fig. 5(b), the Fourier peaks appear at the very middle positions of the positive and negative sides of the spatial frequency axis when the phase difference interval is $\pi / 2$. This property was used to adjust the galvano scanner of Fig. 2 to have the necessary phase interval of $\pi / 2$.

\section{Experimental Result}

To confirm the feasibility of applying the proposed single-step full-range method to biological samples, human skin and tooth were imaged with the bulk setup of Fig. 1. Figures 6(a) $-6(\mathrm{c}), 6(\mathrm{~d})-6(\mathrm{f})$, and 6(g)-6(i) present the SD OCT images of a human finger [27], a fingernail near the nail fold region [14], and a tooth near the dentino-enamel junction region [20], respectively. The figures in the first column are the full-range images obtained using the proposed single-step method, and the ones in the second column were obtained using the BM scan method. The last column shows the corresponding standard SD OCT image, which is overlapped with its complex-conjugate image. We can see that the full-range images, obtained with both the single-step and the BM scan methods, show the clear structure inside the samples. Figures $6(\mathrm{a})$ and $6(\mathrm{~b})$ clearly show the 


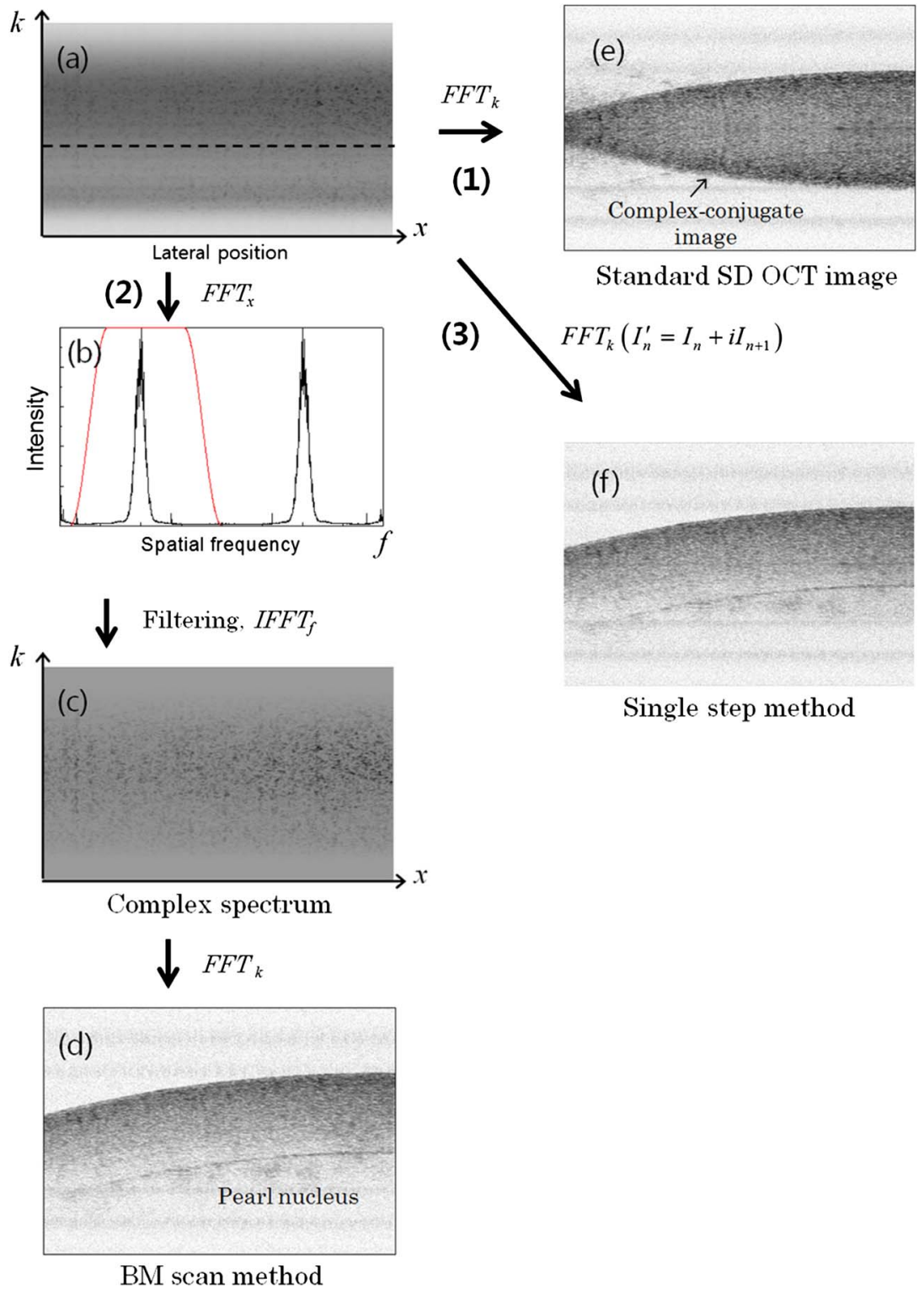

Fig. 5. Flow diagram of full-range image reconstruction process. (1) Standard SD OCT image process, and (2) and (3) full-range methods: the BM scan and the single-step method. (a) 2D spectral interferogram, (b) result of FFT of spectral interferogram in the lateral direction (trapezoid-shape solid line indicates spatial frequency filter), (c) the 2D complex spectral interferogram obtained by applying the process of filtering and inverse FFT to (b) for all wavenumbers, (d) full-range image obtained using the BM scan method after FFT of (c) in the optical wavenumber domain, (e) standard SD OCT image overlapped with a complex-conjugate image, and (f) full-range image using single-step method. It is obtained after a single complex FFT in the wavenumber domain.

interface between epidermis and dermis of the human finger. In Figs. 6(d) and 6(e), we can see the nail plate, epidermis, and dermis of the human fingernail. With Figs. $6(\mathrm{~g})$ and $6(\mathrm{~h})$, the dentino-enamel junction of a human tooth is clearly observed. Each image has a size of $2.7 \mathrm{~mm} \times 3.0 \mathrm{~mm}$ (depth $\times$ lateral:750 pixels $\times 2000$ pixels), and was obtained at an acquisition speed of 10 frames/s.

Process times for the standard, the BM scan, and the single-step method were then compared in order to investigate time efficiency, and summarized in Table 1 . Note that the horizontal and vertical axes represent the applied image processing method and the number of acquired frames, respectively.
The computation times for a single frame were 360,740 , and $470 \mathrm{~ms}$, respectively, which included the rescaling and dispersion compensation processes [28]. The processing time was almost linearly proportional to the number of frames. For 1000 frames, the single-step method took $470 \mathrm{~s}$, which was longer than the standard method by $110 \mathrm{~s}$ but shorter by $260 \mathrm{~s}$ than the BM scan method. The reduction of the computation time by the single-step method with respect to the BM scan method is about $36 \%$. The signal processing was programmed based on the Labview 2011 software. The processing was conducted on an Intel quadcore desktop i7-3770 CPU in a Microsoft Windows 7 with 64 bit environment. 
(a)

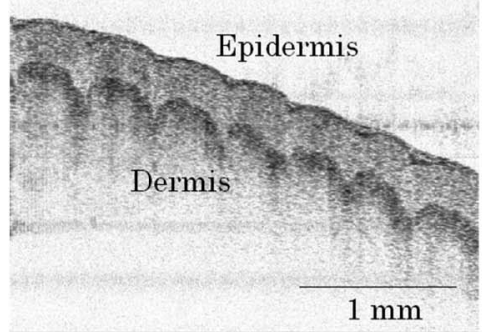

(d)
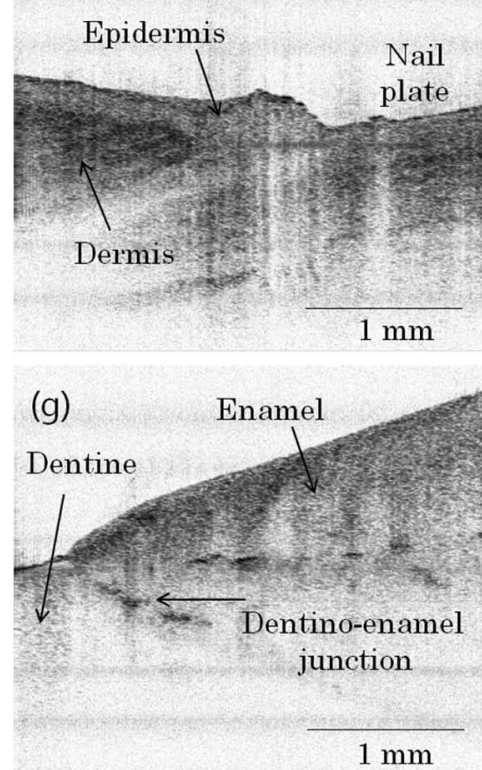

(b)

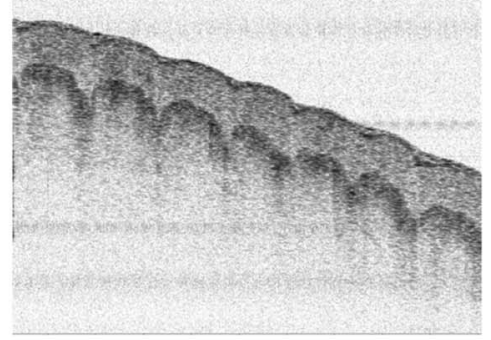

(e)
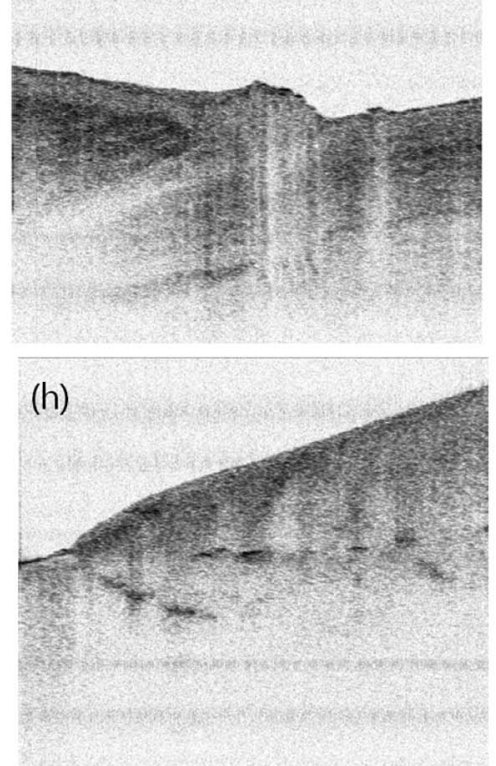

(c)

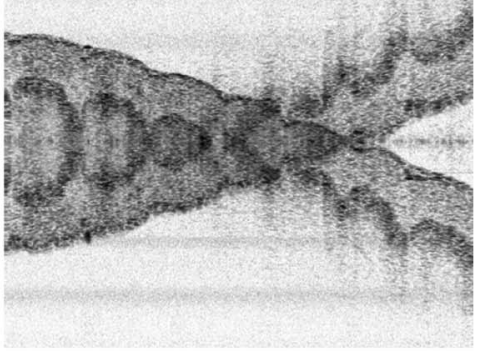

(f)
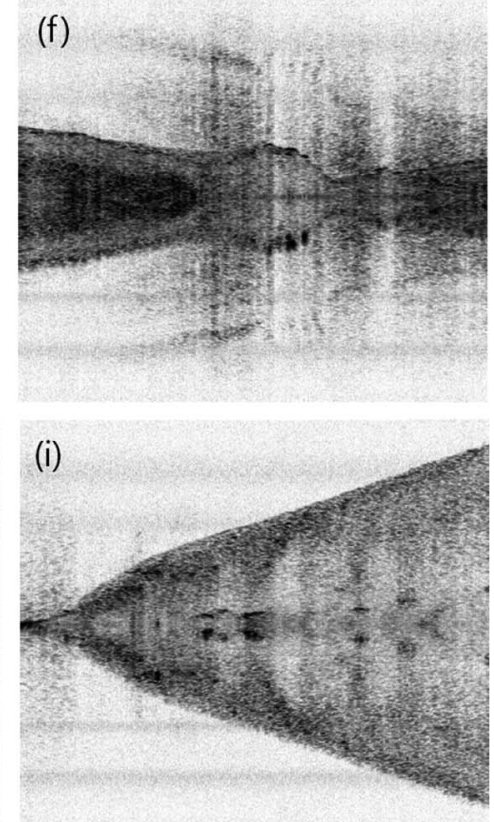

Fig. 6. (a)-(c), (d)-(f), and (g)-(i) are SD OCT images of a human finger, fingernail, and tooth, respectively. The figure in the first column corresponds to a full-range image obtained using the single-step method, the figure in the second column is obtained using the BM scan method, and the full-range image of (a) and (b), (d) and (e), and (g) and (h) shows the clear structure inside of the sample. The last column corresponds to a standard SD OCT image overlapped with a complex-conjugate image.

\section{Single-Step Method in a Fiber-Optic Probe-Based System}

\section{A. Schematic}

After confirming the concept of the single-step method with the bulk-optic-based system, a fiberoptic probe-based system was implemented. A magnetically actuated probe was adapted for the sample scanning because of its low power consumption and low driving voltage [29]. The probe consisted of a solenoid and a single-body lensed fiber, as presented in Fig. 7 . At the middle of the fiber, a small iron-bead was attached for receiving the magnetic force of the solenoid. The single-body lensed fiber was fabricated by forming a lens on the end of the coreless silica fiber that was fusion spliced to the end of a singlemode fiber [(SMF) core/cladding: 5.6/125 $\mu \mathrm{m}$ ], as in Fig. 7(b). The system schematic equipped with the fiber probe is depicted in Fig. 8. The off-axis galvano scanner was used in the reference arm for the phase shifting. In the sample arm, due to the arc motion of the fiber tip, there is an additional phase shift, as illustrated in Fig. 9. When the fiber length is long enough and/or the fiber scanning angle is sufficiently

Table 1. Comparison of Processing Time between the Standard, the BM Scan, and the Single-Step Method

\begin{tabular}{lcccc}
\hline & \multicolumn{3}{c}{ Method } \\
\cline { 2 - 4 } No. of Frames & Standard [s] & BM Scan [s] & Single Step [s] & $\begin{array}{c}\text { Reduction of Computational } \\
\text { Time [\%] }=(B M-S i n g l e) / B M \times 100\end{array}$ \\
\hline 100 & 36 & 74 & 47 & 36.49 \\
500 & 180 & 370 & 240 & 35.14 \\
1000 & 360 & 730 & 470 & 35.62 \\
\hline
\end{tabular}




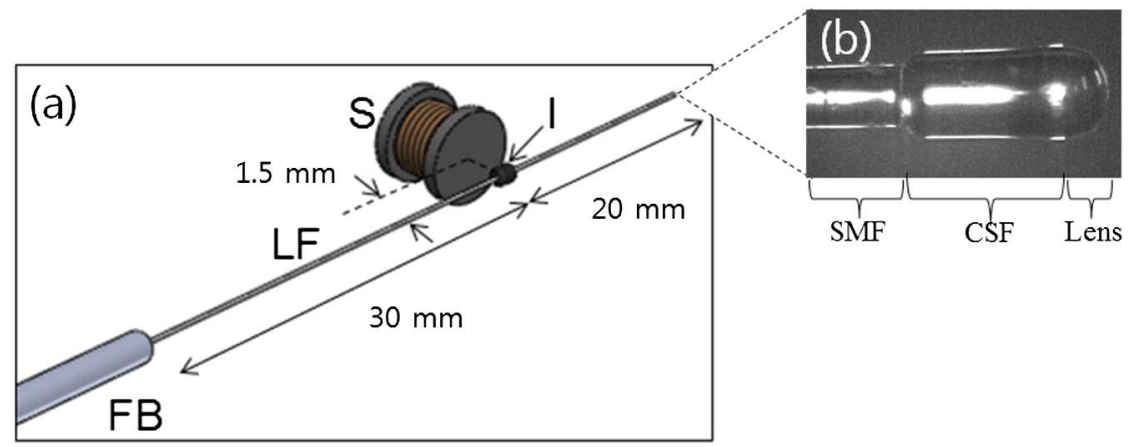

Fig. 7. Schematic of the magnetically actuated probe. (a) The probe is composed of a solenoid and an iron-bead loaded single-body lensed fiber and actuated by magnetic force between an iron-bead and a solenoid. (b) Photograph of the single-body lensed-fiber. S, solenoid; I, iron-bead; LF, lensed fiber; FB, fixed bar; SMF, single-mode fiber; CSF, coreless silica fiber.

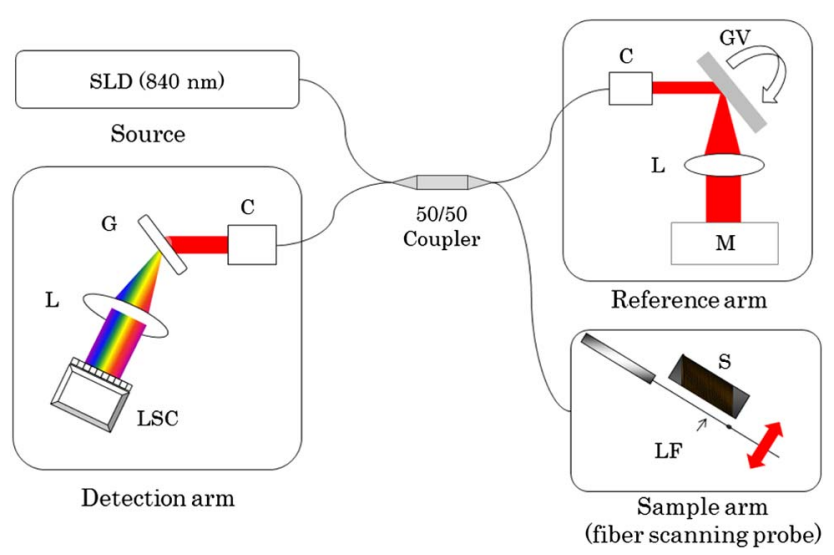

Fig. 8. Schematic of the fiber-optic probe-based full-range SD OCT system. SLD, superluminescent diode; C, collimator lens; M, mirror; L, lens; LSC, line scan CCD camera; G, grating; GV, galvano scanner; S, solenoid; LF, lensed fiber.

small, the additional phase shift can be neglected. However, fortunately, the single-step method only concerns about the phase difference between two neighboring A-lines, not the total phase shift occurring during a B-scan. The lateral resolution of the

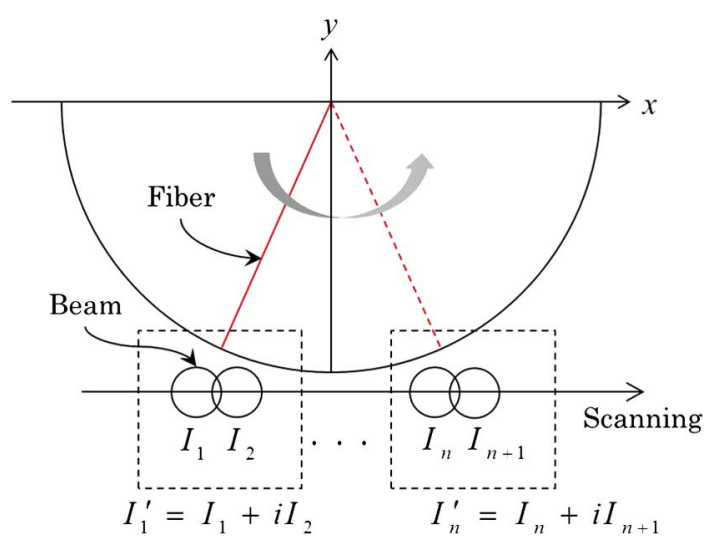

Fig. 9. Illustration showing the concept of suggested single-step method in a fiber-optic probe-based system. $n$, number of A-lines; $I_{n}$ and $I_{n+1}$, two neighboring A-lines at $n$th and $n+1$ th in lateral direction. The motion of fiber is drawn in the rectangular coordinate. The complex spectral interferogram is made with two neighboring A-lines.

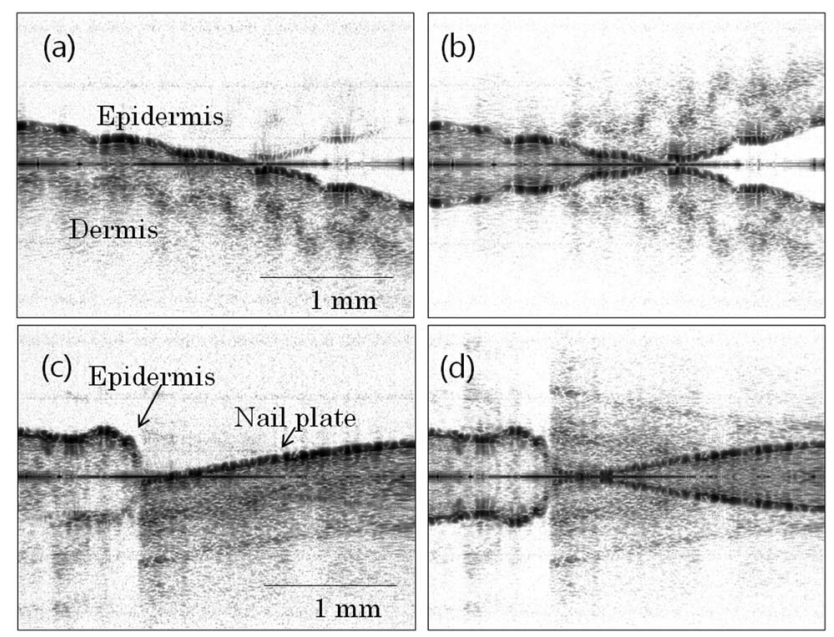

Fig. 10. (a),(b) and (c),(d) are SD OCT images of a human fingertip and fingernail, respectively. The figure in the first column corresponds to the full-range image obtained using the single-step method, the figure in the second column is the standard SD OCT image overlapped with a complex-conjugate image, and the full-range images of (a) and (c) show the clear structure inside of the sample.

probe was $14 \mu \mathrm{m}$ and the oversampling ratio was about 6 .

\section{B. Experimental Result}

Using the implemented fiber scanning probe, as biological specimens, human fingertip and fingernail were imaged and shown in Fig. 10. Figures 10(a) and 10(c) display the full-range SD OCT images obtained with the single-step method, and Figs. 10(b) and $10(\mathrm{~d})$ are the corresponding standard SD $\overline{\text { OCT }}$ images. We can clearly see that the complexconjugate artifact, or the image folding feature, in the standard image is completely disappeared in the image of the single-step method. As mentioned at Section 2.C, however, due to the phase-insensitive DC and autocorrelation signals, we can see rather thick horizontal lines in Figs. 10(a) and 10(c). The scanning speed was 20 frames/s and the image size was $2.3 \mathrm{~mm} \times 3 \mathrm{~mm}$ (depth $\times$ lateral:639 pixels $\times$ 1250 pixels). Since the number of A-lines was altered 
from 2000 to 1250 lines, the galvano scanner in the reference arm was realigned based on Eq. (5) to provide the same phase interval of $\pi / 2$.

\section{Summary and Conclusion}

In summary, we have proposed a single-step method for a fiber-optic probe-based full-range SD OCT system, which has a simple image processing algorithm and a short processing time. The idea for this method was adopted from the two-step phase-shifting method, generally used for a bulk-optic-based system, but appropriately modified for a fiber-optic probe-based system. The phase of each A-line interferogram of SD OCT was modulated, so that the phase difference between two nearest neighboring A-lines became $\pi / 2$. From these two phase-shifted neighbor interferograms, a complex spectral interferogram could be constructed, from which the complex-conjugate-free image could be reconstructed with just a single Fourier transform. For the fiberoptic probe-based system, the sample probe was made in a form of single-body lensed fiber and scanned by a solenoid, but the phase modulation was applied on the reference arm with an off-axis galvano scanner. The complex-conjugate-free, fullrange SD OCT images of some biological samples were achieved and compared with the ones obtained with the standard and the BM scan methods. With the standard method, the image was folded along the zero-phase shift line. In the BM scan method, the processing time was long since it needed three times of FFT processes. With the proposed single-step method, however, we could successfully unfold the image and reduce the processing time by $36 \%$ over the BM scan method. We hope that this study will lead to the development of a high-contrast endoscopic OCT system in near future.

This study was supported by a grant of the Korean Health Technology R\&D Project Ministry for Health, Welfare \& Family Affairs, Republic of Korea (No. A100490) and supported by the Industrial Strategic technology development program funded by the Ministry of Knowledge Economy (MKE, Korea) (No. 10040121).

\section{References}

1. D. Huang, E. A. Swanson, C. P. Lin, J. S. Schuman, W. G. Stinson, W. Chang, M. R. Hee, T. Flotte, K. Gregory, C. A. Puliafito, and J. G. Fujimoto, "Optical coherence tomography," Science 254, 1178-1181 (1991).

2. A. F. Fercher, C. K. Hitzenberger, G. Kamp, and S. Y. El-Zaiat, "Measurement of intraocular distances by backscattering spectral interferometry," Opt. Commun. 117, 43-48 (1995).

3. G. Häusler and M. W. Lindner, “"Coherence radar" and "spectral radar"-new tools for dermatological diagnosis," J. Biomed. Opt. 3, 21-31 (1998).

4. R. Leitgeb, C. K. Hitzenberger, and A. F. Fercher, "Performance of Fourier domain versus time domain optical coherence tomography," Opt. Express 11, 889-894 (2003).

5. J. Schmit and K. Creath, "Extended averaging technique for derivation of error-compensating algorithms in phase-shifting interferometry," Appl. Opt. 34, 3610-3619 (1995).
6. X. F. Meng, L. Z. Cai, X. F. Xu, X. L. Yang, X. X. Shen, G. Y. Dong, and Y. R. Wang, "Two-step phase-shifting interferometry and its application in image encryption," Opt. Lett. 31, 1414-1416 (2006).

7. Y. Awatsuji, T. Tahara, A. Kaneko, T. Koyama, K. Nishio, S. Ura, T. Kubota, and O. Matoba, "Parallel two-step phaseshifting digital holography," Appl. Opt. 47, D183-D189 (2008).

8. M. Wojtkowski, A. Kowalczyk, R. Leitgeb, and A. F. Fercher, "Full range complex spectral optical coherence tomography technique in eye imaging," Opt. Lett. 27, 1415-1417 (2002).

9. R. A. Leitgeb, C. K. Hitzenberger, A. F. Fercher, and T. Bajraszewski, "Phase-shifting algorithm to achieve highspeed long-depth-range probing by frequency-domain optical coherence tomography," Opt. Lett. 28, 2201-2203 (2003).

10. E. Götzinger, M. Pircher, R. A. Leitgeb, and C. K. Hitzenberger, "High speed full range complex spectral domain optical coherence tomography," Opt. Express 13, 583-594 (2005).

11. S. Zotter, M. Pircher, E. Götzinger, T. Torzicky, M. Bonesi, and C. K. Hitzenberger, "Sample motion-insensitive, full-range, complex, spectral-domain optical-coherence tomography," Opt. Lett. 35, 3913-3915 (2010).

12. M. V. Sarunic, M. A. Choma, C. Yang, and J. A. Izatt, "Instantaneous complex conjugate resolved spectral domain and swept-source OCT using $3 \times 3$ fiber couplers," Opt. Express 13, 957-967 (2005).

13. Y. Yasuno, S. Makita, T. Endo, G. Aoki, M. Itoh, and T. Yatagai, "Simultaneous B-M-mode scanning method for real-time fullrange Fourier domain optical coherence tomography," Appl. Opt. 45, 1861-1865 (2006).

14. R. K. Wang, "In vivo full range complex Fourier domain optical coherence tomography," Appl. Phys. Lett. 90, 054103 (2007).

15. C. Wu, T. Chi, C. Lee, Y. Kiang, C. Yang, and C. Chiang, "Method for suppressing the mirror image in Fourier-domain optical coherence tomography," Opt. Lett. 36, 2889-2891 (2011).

16. R. A. Leitgeb, R. Michaely, T. Lasser, and S. C. Sekhar, "Complex ambiguity-free Fourier domain optical coherence tomography through transverse scanning," Opt. Lett. 32, 3453-3455 (2007).

17. B. Baumann, M. Pircher, E. Götzinger, and C. K. Hitzenberger, "Full range complex spectral domain optical coherence tomography without additional phase shifters," Opt. Express 15, 13375-13387 (2007).

18. L. An and R. K. Wang, "Use of a scanner to modulate spatial interferograms for in vivo full-range Fourier-domain optical coherence tomography," Opt. Lett. 32, 3423-3425 (2007).

19. K. Zhang, Y. Huang, and J. U. Kang, "Full-range Fourier domain optical coherence tomography imaging probe with a magnetic-driven resonant fiber cantilever," Opt. Eng. 50, 119002 (2011).

20. E. J. Min, J. G. Shin, J. H. Lee, Y. Yasuno, and B. H. Lee, "Full range spectral domain optical coherence tomography using a fiber-optic probe as a self-phase shifter," Opt. Lett. 37, 3105-3107 (2012).

21. Z. Yaqoob, J. Wu, E. J. McDowell, X. Heng, and C. Yang, "Methods and application areas of endoscopic optical coherence tomography," J. Biomed. Opt. 11, 063001 (2006).

22. A. Dubois, L. Vabre, A. Boccara, and E. Beaurepaire, "Highresolution full-field optical coherence tomography with a Linnik microscope," Appl. Opt. 41, 805-812 (2002).

23. S. Zotter, M. Pircher, T. Torzicky, M. Bonesi, E. Götzinger, R. A. Leitgeb, and C. K. Hitzenberger, "Visualization of microvasculature by dual-beam phase-resolved Doppler optical coherence tomography," Opt. Express 19, 1217-1227 (2011).

24. B. J. Vakoc, S. H. Yun, J. F. de Boer, G. J. Tearney, and B. E. Bouma, "Phase-resolved optical frequency domain imaging," Opt. Express 13, 5483-5493 (2005).

25. H. C. Hendargo, R. P. McNabb, A. Dhalla, N. Shepherd, and J. A. Izatt, "Doppler velocity detection limitations in spectrometer-based versus swept-source optical coherence tomography," Biomed. Opt. Express 2, 2175-2188 (2011). 
26. M. J. Ju, S. J. Lee, E. J. Min, Y. Kim, H. Y. Kim, and B. H. Lee, "Evaluating and identifying pearls and their nuclei by using optical coherence tomography," Opt. Express 18, 13468-13477 (2010).

27. X. Liu, Z. Lu, R. Lewis, M. J. Carré, and S. J. Matcher, "Feasibility of using optical coherence tomography to study the influence of skin structure on finger friction," Tribol. Int. 63, 34-44 (2013).
28. S. Makita, T. Fabritius, and Y. Yasuno, "Full-range, highspeed, high-resolution $1 \mu \mathrm{m}$ spectral-domain optical coherence tomography using BM-scan for volumetric imaging of the human posterior eye," Opt. Express 16, 8406-8420 (2008).

29. H. P. Hu, K. D. Le, and J.-C. Chiao, "An optical scanner based on electromagnetically actuated optical fiber," Proc. SPIE 6109, 610906 (2006). 